\title{
Un nouvel Institut de Myologie en Floride
}

\author{
Gillian Butler-Browne
}

La mission de l'Institut de Myologie de l'Université de Floride est de découvrir les causes et la physiopathologie des maladies neuromusculaires, de mener des études précliniques pour évaluer des thérapies innovantes, de conduire des essais cliniques pour ces maladies et de réunir les chercheurs et cliniciens de l'Université de Floride, mais aussi d'autres institutions qui veulent comprendre les bases de la biologie et de la physiopathologie de ces atteintes et développer des traitements.

L'Institut de Myologie de l'Université de Floride (UF) a été créé il y a un an, et est actuellement dirigé par le Dr H. Lee Sweeney, un chercheur de réputation mondiale dans le domaine des maladies neuromusculaires (MNM). Les recherches du Dr Sweeney sont principalement centrées sur les myosines, " moteurs" moléculaires de la cellule musculaire, les maladies cardiovasculaires d'origine génétique, les aspects cardiaques et squelettiques des dystrophies musculaires, ainsi que le développement de traitements pour les MNM à l'aide de petites molécules. L'Institut de Myologie de l'UF n'est pas regroupé dans un seul bâtiment, mais il rassemble des chercheurs répartis sur le campus de l'UF avec des industries privées et d'autres institutions dans le but de créer un effort collaboratif pour mieux comprendre et traiter les MNM. Les activités de l'Institut de Myologie de l'UF concernent la recherche fondamentale sur les muscles squelettiques, cardiaques et

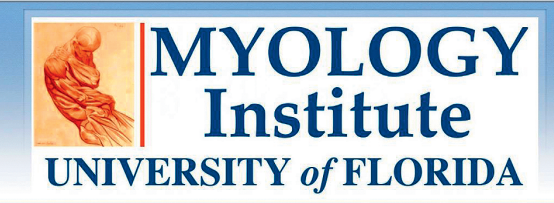

développement de la fibrose et l'infiltration graisseuse. Cette labellisation a été suivie en octobre 2015 par un soutien financier du NIH (National Institutes of Health) à hauteur de 10,76 millions de dollars pour développer un projet multidisciplinaire portant sur la dégénérescence musculaire en situation pathologique.

Une valeur ajoutée à cet Institut est sa proximité avec les hôpitaux locaux, l'école de médecine et l'école vétérinaire. Les efforts sont actuellement développés pour multiplier les essais cliniques pour les dystrophies musculaires, et l'Institut développe une expertise mondiale dans l'utilisation de l'IRM dans le suivi des patients souffrant de Dystrophie Musculaire de Duchenne (DMD). Ainsi, dans une étude multicentrique financée par une subvention du NIH de 7,5 millions de dollars dont l'investigateur principal est Krista Vandenborne, les chercheurs de l'UF ont montré que la technologie IRM lisses, et la recherche translationnelle impliquant des modèles cellulaires et animaux des MNM et cardiovasculaires. L'Institut a pour mission de faciliter les études et les essais cliniques dans ces domaines, ainsi que d'assurer la formation, d'organiser des conférences nationales et internationales dans ces domaines, et enfin de mettre en place des plateformes performantes pour aider à accomplir ces missions.

Gillian Butler-Browne Centre de Recherche en Myologie UMRS974- UPMC Sorbonne

Universités/U974

- Inserm/FRE3617

CNRS/AIM

Groupe hospitalier

Pitié-Salpêtrière, Paris,

France

Contact

gillian.butler-browne@ upmc.fr
L'Institut comprend actuellement 33 chercheurs confirmés, et a recruté récemment comme directrice adjointe Karyn Essen, une chercheuse fondamentale professeur de Physiologie et de Génomique fonctionnelle. Au cours de sa première année d'existence, l'Institut est progressivement monté en puissance avec la labellisation de "Senator Paul Wellstone Muscular Dystrophy Cooperative Research Centre ", et pour mission d'améliorer la régénération dans les dystrophies musculaires en inhibant le permet des mesures précises et non invasives sur les membres inférieurs des enfants souffrant de DMD. L'IRM permet de suivre rapidement les effets de thérapies innovantes dans les essais cliniques, permettant ainsi aux chercheurs de détecter des changements au niveau musculaire en seulement quelques mois. Dans une nouvelle étude, les Drs Vandenborne, Sweeney, et Maren et d'autres membres de l'équipe vont suivre la progression de la DMD chez 136 patients ayant déjà participé à l'étude princeps d'imagerie, et les chercheurs vont aussi tester l'efficacité de l'IRM pour l'évaluation des membres supérieurs. Si les résultats obtenus montrent une sensibilité égale sur les membres supérieurs, cela permettra d'accroître de manière significative le nombre d'enfants DMD pouvant rentrer dans des essais cliniques, souvent limités jusqu'alors à des patients ambulants. Le but final de l'étude est de développer l'imagerie par IRM comme biomarqueur 
pouvant être utilisé chez des enfants non ambulants, et donc d'accroître les inclusions dans les études cliniques.

L'Institut de Myologie de l'Université de Floride, bien qu'encore jeune, présente déjà de nombreux atouts pour devenir un acteur majeur dans la Myologie internationale, et il a déjà développé des contacts avec d'autres Instituts internationaux : ainsi des discussions fructueuses ont déjà été menées avec
l'Institut de Myologie de la Pitié-Salpêtrière, en attendant la signature d'accords entre les tutelles qui viendront formaliser cette coopération internationale au plus haut niveau scientifique.

A novel Myology Institute in Florida

\section{LIENS D'INTÉRÊT}

L'auteur déclare n'avoir aucun lien d'intérêt concernant les données publiées dans cet article. 